\title{
An Ultrasonic Piezoelectric Power Generator for Public and Industrial Buildings Application
}

\author{
Daniel FLOYD ${ }^{1} \&$ Mahmoud SHAFIK ${ }^{2}$ \\ University of Derby, Markeaton Street, Derby DE22 3AW, UK
}

\begin{abstract}
This paper presents an ultrasonic micro power generator using the piezoelectric direct effect phenomena. The micro power generator consists of 2 main elements, a movement matt including PZT elements and an energy harvesting circuit. The movement matt is made up of a four PZT elements each element creating a cantilever beam. The energy harvesting circuit is made up of an LTC3588 Evaluation Board and an LDR night light. Computer simulation and modelling using finite element analysis for the proposed generation method is discussed and used in the design and development process. Finite element analysis has been used to evaluate the PZT structure by performing an algebraic solution of a set of equations, describing an ideal model structure, with a finite number of variables. The simulation and modelling enabled to select the material and best method of operation. A prototype of the proposed generator was built and tested. This demonstrated that piezoelectric material could produce up to $36 \mathrm{~V}$, although the overall impedance of such devices was shown to be linear depending on the force applied with an average of $36 \mathrm{M} \Omega$. The Energy harvesting circuit allowed an output super capacitor to be step charged taking an average time of 35-minuites to charge and 2-minutes to discharge through the selected load.
\end{abstract}

Keywords: Kinetic Energy, Direct Piezo Effect, Energy Harvesting, LTC3588, Piezoelectric generator

\section{Introduction}

Piezoelectric was discovered in 1880 when Pierre Currie and Jacques realised that certain crystalline material such as ceramic and when subjected to a mechanical force or stress became electrically charged this known today as the 'Direct Piezo Electric Effect.' With more experiments Pierre began to realise that when an electrical field was placed across a piezoelectric material it began to lengthen or shorten according to the polarity, this is known as the 'Converse Electric Effect'. Piezoelectric material has been subjected to many kinds of tests and experiments and is used today in many applications. Piezo is formed from certain solid structures such as crystal although in more modern techniques a manmade substance of Lead, Zirconate Titanate (PZT) are used. PZT materials have been formed they contain a positive and negative charge known as an electrical dipole. These electrical dipoles are formed in groups known as Weis Domains which are scattered with no orientation thus little operating characteristics. These undergo a

\footnotetext{
${ }^{1}$ Daniel Floyd. D.floyd@hotmail.co.uk

${ }^{2}$ Mahmoud Shafik.mshafik@derby.ac.uk
} 
polarisation exercise which is done by exposing the element to temperatures at just below the 'Curie Point' and subjecting this to strong electric field, causes the randomly orientated dipoles to become orientated in the same direction as the electric field. The electrical dipoles become polarised due to the electric field acting upon these defined as electric dipole moment. After the electric field has been placed across this material the dipoles in their 'Weiss Domains' have the ability to hold their alignment, known as the remnant polarization or hysteresis.

The mathematical model of the polarization is shown as equation 1 , denotes where $\mathrm{P}$ is the polarization measured in $\mu C / \mathrm{cm}^{2}$, electrical displacement denoted as $\mathrm{D}, \epsilon^{t}$ is the permittivity relating to the compressive stress applied and $E$ is the electric field applied.

$$
\mathrm{P}=D-\epsilon^{t} \times E(1)
$$

When a voltage is applied across a Piezo material this causes deflection in the ceramic plate. The deflection is proportional to the voltage applied and follows characteristics demonstrated by the butterfly loop. This effect is known as the 'Inverse Piezo effect' and can be used for applications such as motors, actuators, and transducers. Conversely to this when an external stress or pressure is applied to the PZT formation the dipole moment is changed thus causing an electrical voltage to be generated. The generated voltage is directly proportional to the applied stress known as the 'Direct Piezo Effect'. This energy generated can be used for applications such as sensors, musical pickups (microphones) and for the as this paper notes can be used for energy harvesting. Connecting a Piezo element can be done in a closed loop which shorts the material thus causing the material to become depolarised, this is used for applications where a high voltage is needed but only as a one off, open loop is where the PZT is connected through a load or other device and the polarization remains. The PZT cut into a flat plate used as a cantilever beam has internal resistance, inductance, and capacitance (reactance). Figure 1 illustrates the equivalent Circuit for an open circuit model. Because of the series connected impedance the total is calculated in $\mathrm{R}+\mathrm{JX}$ form where $\mathrm{XC}$ is equal to (capacitance) is $\frac{1}{2 \pi F C}$ and $\mathrm{XL}$ inductance is $2 \pi F L$.

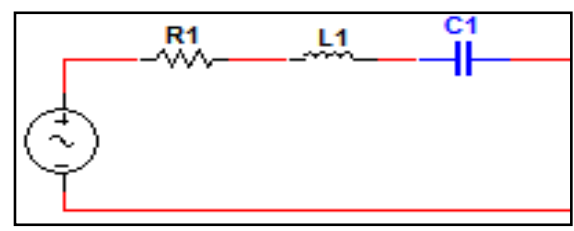

Figure 1. PZT Equivalent Circuit.

The PZT constants have been used for the various calculations below. Which have been conducted for the direction as stated in the equation illustrated in bold. These equations have been calculated using a human's weight of $83 \mathrm{~kg}$.

$$
\begin{aligned}
& \mathbf{V}=\frac{\mathbf{3} \mathbf{g} 31 \mathbf{F}_{3} \mathbf{H}}{\boldsymbol{L} \boldsymbol{W}}=\frac{3 *\left(10 \times 10^{-3}\right) * 804 * 50}{4 * 32 * 0.52}=36.23 \mathrm{~V} \\
& \mathbf{Q}=\boldsymbol{d}_{33} \boldsymbol{x} \boldsymbol{T}=820 \times 10-12 \times 244.97=200.87 n C \\
& \boldsymbol{I}=\frac{\boldsymbol{q}}{\boldsymbol{t}}=\frac{200.87 \mathrm{nC}}{206.185 \times 10-3}=975 \mathrm{nA}
\end{aligned}
$$




$$
\begin{aligned}
& \boldsymbol{Z t}=\frac{\boldsymbol{V}}{\boldsymbol{I}}=\frac{36 \mathrm{~V}}{975 \mathrm{nA}}=36 \mathrm{M} \Omega \\
& \boldsymbol{P}=\boldsymbol{V} \boldsymbol{x} \boldsymbol{I}=36 \mathrm{~V} \times 975 \mathrm{nA}=35.1 \mu \mathrm{W}
\end{aligned}
$$

The calculations show that the impedance of the material is susceptible to the voltage generated. The output voltage is linearly proportional to the applied force, therefore the applied force on the PZT plate is also proportional to the impedance. The quiescent current or current drawn whilst under no load is inversely proportional to the impedance (Ohms Law) thus generates more current upon a higher force being applied.

\section{PZT Plats Finite Element Analysis}

Finite element analysis is a technique used to analyse a specific design or model when placed under stresses or strains. FEA is usually used within manufacturing, mechanical engineering or civil engineers to measure the strength parameters of items such as a bridge. There are two forms of modelling 2D which gives less accurate results and 3D which gives more accurate results although to model in 3D a high-end computer is needed. Using this technique allows engineers to input various algorithms measuring either the linearity or non-linearity of the material, these systems can test the material right the way to the point of fracture which is ideal for civil engineers wanting to know the load points on a bridge and altering any problems to strengthen the bridge before the build begins. Comsoll Multiphysics has been supplied on a trial basis with the MEMS (Micro Electrical Mechanical Systems) material library package. PZT 507 materials characteristic are not loaded into this package. Unfortunately to program new materials in Comsoll various parameters were needed in which were not added into the data sheet and upon speaking to Morgan Ceramics these were not able to be obtained due to the complex and expensive testing procedures. The PZT5H has been used because when the various materials in Comsoll MEMS package were compared this was the closest match.

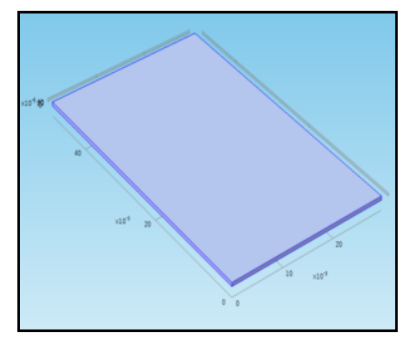

Figure 2. 3D Model of Piezoelectric Plates

To model the Piezoelectric plates accurately the measurements must be correct and therefore the dimensions were the same to that of the PZT507 Plates ordered from Morgan Technical Ceramics of 30x50x0.52mm (W,D,H). This has been completed using a high-end computer as the 3D Multiphysics model figure 2 above shows. Upon building this a tetrahedral mesh is created which consists of 6,585 different tetrahedral elements or geometry's. A Tetrahedron is a three-dimensional geometry shape which comprises of four triangles each joined by vertices. This shape is more commonly known as a 
triangular pyramid. Comsoll splits the 3D models up into small nodes these nodes are what form the structure of tetrahedral mesh. The mesh contains the information of the material characteristics in matrices format which then allows the computer to simulate the different reactions, acting upon the model in the various directions. Once our mesh had been created, we then configured our model to have a fixed constraint or fixed point. The fixed point defines the boundary of which is static allowing the rest of the material to be flexed, for the purpose of this analysis tests have been conducted using different fixed constraints such as boundary 1 which demonstrates a cantilever beam and boundary 3 as another which demonstrating the PZT as a flat plate. Boundary 3 has also been selected as an electrical ground which is connected to our external circuit as the neutral electrode. 480 Newton's is equal to 10 stone which is the selected weight used for the purpose of these experiments and corresponds with the mathematical analysis section. Using the various parameters of the PZT material and the mapped mesh Comsoll was then set to perform an automated analysis on the deformation of the material for the two different fixed constraints modelling the flat plate method and the cantilever beam method illustrated below.

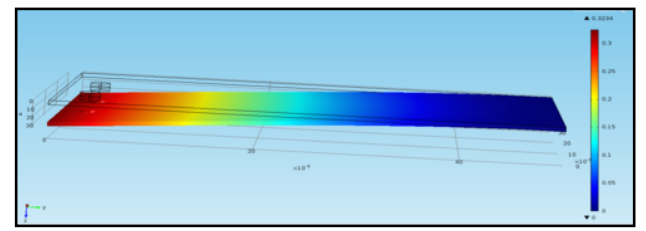

Figure 3. Deformation of Piezoelectric Plates

Figure 3 illustrates that whilst subjecting the PZT material to 480 Newton's of force the material was deformed by a maximum of $0.3 \mathrm{~cm}$ illustrated in red. It is noted that the material is deformed the most on the tip of the PZT plate this is because of the cantilever beam effect. This demonstrates that the PZT plate will be able to withstand the applied force and upon the PZT reaching the maximum applied force a clear fracture would be shown within the light blue region. Secondary to this cantilever beam the direct effect was simulated which showed with a load applied to the surface of 480 Newton's The Plate had a total displacement factor of $14^{x 10-9} \mathrm{~cm}$ across the top surface.

PZT material and its generating characteristics depends on a specific load applied along with the direction in which the load is applied. Within the FEA analysis a few functions have been created which average out the pressure and displacement applied upon the material causing the output value to be nondependent on this. A variable was created to define the applied load and the simulation was programmed to run the simulation in steps, for each of the variables in order known as a parametric sweep this was then programmed to plot the corresponding results to a $1 \mathrm{D}$ plot group, as shown in figure 4. 


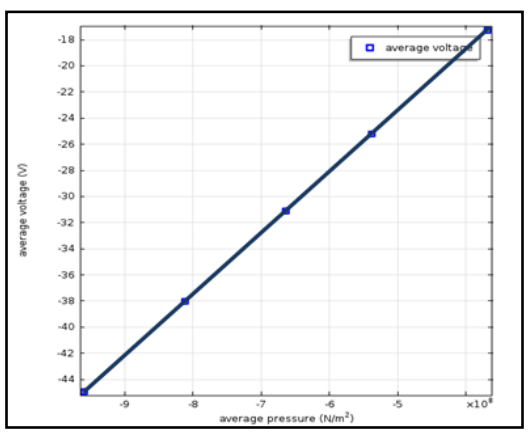

Figure 4. Voltage Vs Pressure

This demonstrates a voltage of $-33 \mathrm{~V}$ being generated with an applied load of $804 \mathrm{~N}$ this is a negative voltage due to the direction of which the pressure is applied therefore if we were to reverse the direction of pressure the voltage would be positive although this simulation implies a difference of $3.23 \mathrm{~V}$ from the manual calculations. This is due to the material parameter differences and given the correct material programmes these measurements would be more accurate, which demonstrates that the mathematical equations taken from APC Piezoelectric Ceramics publication are of correct form for the PZT Cantilever beam. The FEA has allows us to critically analyse and demonstrate that our research into Piezoelectric plates used in the direct effect is 'FACT' thus allowing us to progress onto developing an energy harvesting circuit to demonstrate the Piezo energy cantilever beam generator.

\section{PZT Voltage Harvesting Prototype Experimental Testing, Results and Discussion}

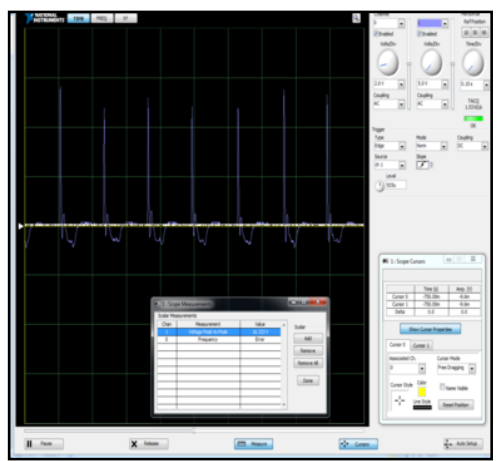

Figure 5. Direct Pressure

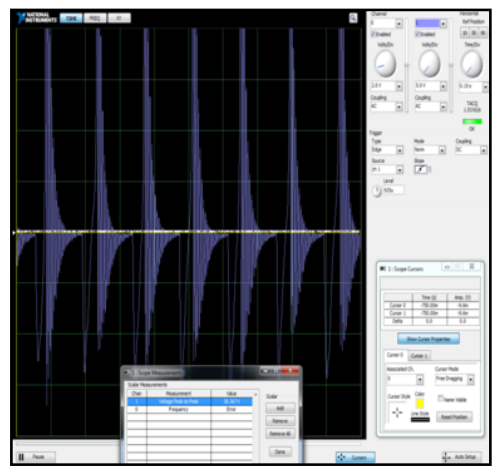

Figure 6. Cantilever Beam

Figures 5 and 6 show the different methods of voltage generation using the cantilever beam right and the direct pressure method left. A maximum voltage of $16 \mathrm{~V}$ and $36 \mathrm{~V}$ as shown below which generate such sudden high peaks because it's at this point in time when the highest amount of deformation is caused. The PZT material like that of a pendulum then returns back to its equilibrium point it does this by gravity 
dampening the oscillating, this oscillating is caused by the restoring force and the mass on the plates. The dipole moment is forced to change in increments as the oscillations dampen thus causing an $\mathrm{AC}$ voltage. On the other hand, the PZT with a fixed constraint as the bottom surface causes little oscillations but a high initial voltage peak. PZT507 as explained has a very high reactance which means that one element on its own produces low power. To overcome this, 4 PZT 507 plates have been connected in parallel known as an array where $\mathrm{VO}$ is the output connections. Figure 7 illustrates the circuit derived for this application. Impedance denoted as $\mathrm{Z}$ is the complex form of resistances which takes phase and magnitude into account. The calculation for a single PZT plate is shown in equation below where the total circuit impedance when calculated in parallel demonstrates the use for the product over sum method.

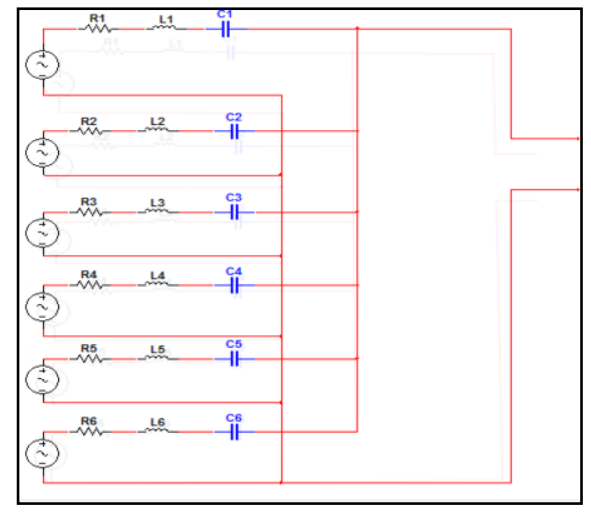

Figure 7. Parallel Array

$$
\begin{aligned}
& \mathrm{ZT}=\frac{1}{\frac{1}{z 1}+\frac{1}{z 2}+\frac{1}{z 3}+\frac{1}{z 4}+\frac{1}{z 5}+\frac{1}{z 6}} \\
& \frac{1}{\frac{1}{36 M}+\frac{1}{36 M}+\frac{1}{36 M}+\frac{1}{36 M}}=9 M \Omega
\end{aligned}
$$

The PZT produces an AC wave form and to convert this voltage to DC a bridge rectifier is used. This consists of 4 diodes placed in a bridge format which are placed so that upon the positive wave form the diode D8 and D7 conduct whilst D5 and D6 don't. This then reverses thus the load always receiving the positive half of the $\mathrm{AC}$ wave form. The Conversion of AC - DC uses the area of the positive cycle A1 and the area of the negative cycle A2 and takes an average value according to the total time period this gives us a Voltage DC (VDC) Value, which is directly due to the output voltage assuming no losses in the circuit. Equation below demonstrates the formula for the areas which is then derived using integration into the final formula as below.

$$
\begin{aligned}
& \text { DC voltage }=\frac{\text { Area } A 1+\text { Area } A 2}{T}=\frac{1}{\pi} \int_{0}^{\pi} \sqrt{2 V S \sin (\omega t) d \omega t} \\
& V D C=\frac{2 x \sqrt{2}}{\pi} \times V S-\mathrm{VD}
\end{aligned}
$$


The Diodes of this circuit have substantial voltage drops which can be found in the manufactures data sheet and is subtracted from the VDC value to give the Total Vout. A buck regulator steps voltage down similar to that of a linear voltage regulator although DC - DC Choppers are $80 \%$ or more efficient. These systems are currently used in many renewable energy harvesting circuits.

$\mathrm{D}=\frac{T o n}{T s}$

$\mathrm{Vo}_{\mathrm{o}}=D \times V d$

Where D is the duty cycle, Ton is the on-time of the MOSFET or transistor, Ts is the switching frequency in seconds and Vs is the supply voltage. The freewheeling diode of a standard chopper contributes to power loss thus reducing the efficiency. For applications such as Piezo energy harvesting the power loss of such circuit is important therefore a synchronous buck regulator has been used. This circuit differs because instead of using a freewheeling diode a $2^{\text {nd }}$ MOSFET or transistor is used thus less power being lost. The Switching controls (PWM) must be alternately switched otherwise when both Q1 and Q2 switches are on at the same time the circuit would be shorted to ground.

Under Voltage Lock Out (UVLO) is a device used whose sole operation is to measures the voltage across the charging capacitor and will not allow any voltage to flow onto the load until the input is above a specified voltage. The UVLO continues to monitor the circuit so as if the input voltage drops below the specified voltage the UVLO transistor will be forced closed due to the rated Zenner diode connected to the gate of the transistor causing no voltage to flow to the output.

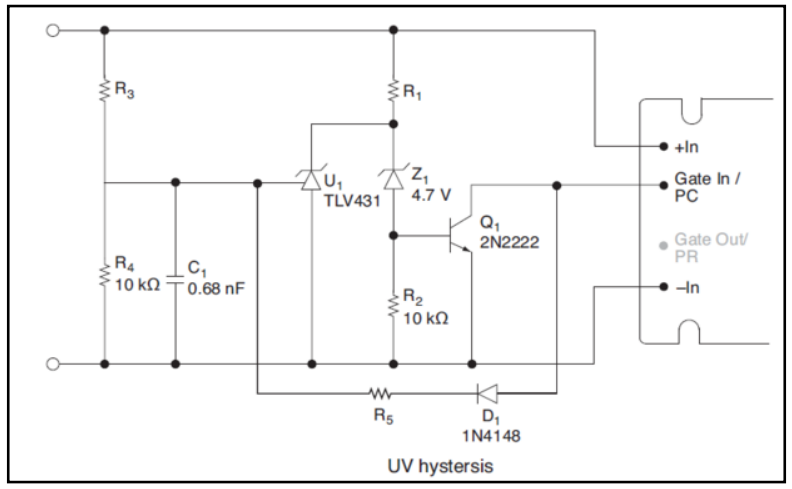

Figure 8. UVLO

Reference: Vicor. (2013)

A varying input voltage like that of a rapidly changing and discharging input capacitor causes such system to be forced into an undesired state of switching. Hysteresis causes a window where such voltage must be above that of the specified voltage + the hysteresis window before the circuit will conduct and will only seize conduction again upon the voltage dropping below specified voltage. Figure 8 illustrates the circuit diagram of a UVLO system. The operation this circuit simply works by Q1 only firing (allowing voltage to pass) when $\mathrm{C} 1$ is charged above that of rated voltage of $\mathrm{U} 1$ and $\mathrm{Z} 1$. If the input drops below the specified voltage $\mathrm{C} 1$ holds some of the charge but discharges 
slowly through R5 thus hysteresis window and therefore when $\mathrm{C} 1$ drops below the U1 and $\mathrm{Z1}$ value, the circuit seizes conduction. The UVLO above has a under voltage of 4.7V a hysteresis window of 500mV taken from the facts on Vicorpower. rev6 (2013).

The LTC3588 semiconductor chip (figure 9) sourced from Linear Technology has a (1) bridge rectification circuit, (2) UVLO, (3) buck convertor, (4) Over current protection, 5 topside NFET (field Effect Transistor) and (6) bottom side PFET as illustrated in the block diagram below. The operation of such circuit is when a charge is generated from the piezo elements the rectifier converts to DC used to charge an input charging capacitor. The UVLO regulates the voltage of the input capacitor and operates the choppers control thus when the input voltage is above $3.5 \mathrm{~V}$ the PFET is switched on which steps the current to $250 \mathrm{~mA}$ and allows flow to the load. After this the charge on the input capacitor dissipates so the UVLO switches PFET off and the NFET on disabling the circuit at which point the LTC3588 enters sleep mode which has minimal power loss, thus allowing the charge to re-accumulate on the input capacitor before the operation repeats.

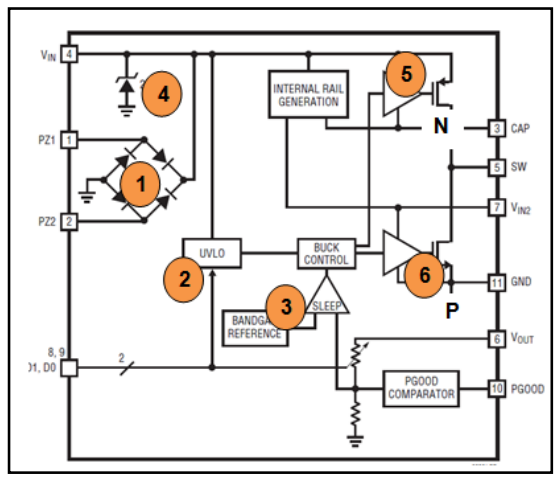

Figure 9. LTC3588

Reference: Linear Technology

LTC3588 - $(10,03,2013)$

The LTC3588 Semiconductor chip is only available in $3 \mathrm{~mm} \times 3 \mathrm{~mm}$ surface mount (SMD) and although the University of Derby have the facilities to mount such device it was decided due to the time constraints the LTC 3588 EMSE-1 evaluation board by linear technology for the purpose of demonstrating such circuitry. Testing and demonstration of the evaluation board is shown on later sections of this paper. The full circuit schematic shown in figure 10 illustrates how a prototype has been built.

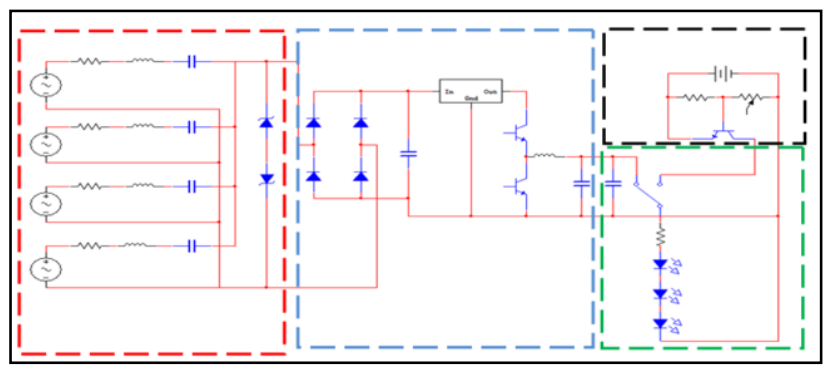

Figure 10. Circuit Diagram

Reference: vicorpower.rev 6 (2013) 
Using the movement board and the full working prototype two tests have been conducted, charging of the super capacitor (SP) and discharging of the SP. The Analysis has been completed using the same weighted humans of 10 stone although the speeds of the tests differed slightly due to the impossibilities of running at the same speed. The results from our testing procedure have been shown below and analysed graphically. Figure 11 illustrates the time taken to charge the 0.1F SP using the EH circuit which looks like similar to an exponential growth, due to the time scale used this is in fact step charged due to the LTC3588'S UVLO switching on and off. The oscilloscope measurements if monitored over a half an hour which is not possible on the University of Derby's equipment. Figure 12 illustrates an exponential discharge for the capacitor. This decay is because of the RC circuit which shows a steeper curve at the beginning; because the discharge rate is faster than starts smoothing off at the point the capacitor loses its charge.

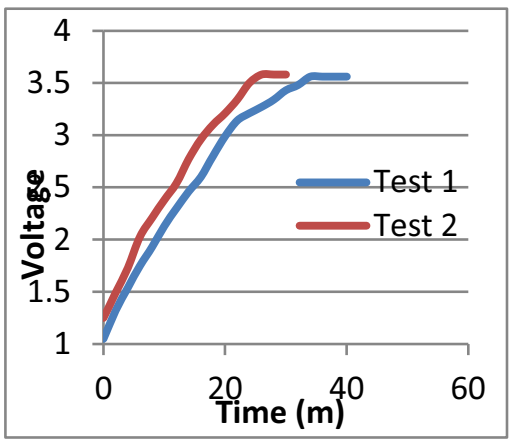

Figure 11. Charge

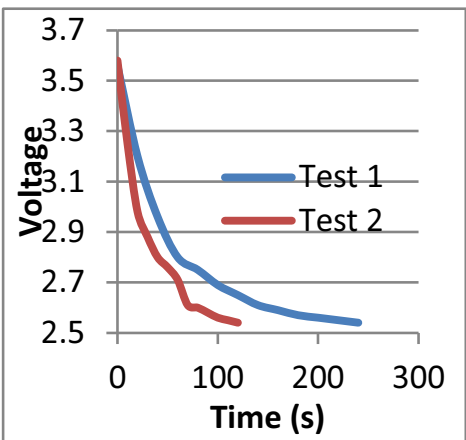

Figure 12. Discharge

\section{Conclusion}

An ultrasonic micro power generator using the piezoelectric direct effect phenomena has been developed and presented in this paper. The micro power generation is generating power on small scales for applications such as portable devices, L.E.D torches etc. Comsoll analysis is used to perform a finite element analysis. The results show that the cantilever beam works better for energy generation than the direct pressure method illustrated through the deformation factor. Further physical testing has then been conducted using the chosen material obtained from Morgan Technical Ceramics proving that that the applied stress is directly proportional to the generated voltage and that the Piezo generated 16V (direct pressure) and 36V (cantilever beam) therefore illustrating the deformation is greater under the cantilever beam, thus this being the best method which was then used for the energy harvesting application. Numerous Piezo Plates connected in an array configuration has been used to step charge an input capacitor through a bridge rectifier. The UVLO monitors the voltage across the capacitor and allows power flow whilst the capacitor is charged $250 \mathrm{mV}$ above the set input voltage. A super capacitor was placed in parallel with this to stabilise the power giving a constant voltage, this therefore illustrates that the circuit could be used for applications such as charging portable device and could be implemented into the rubber matting on running machines or skipping boards. Although the aims and objectives of this applied research 
work have been successfully met, research is needed to enable higher efficiency and optimization of the PZT materials. The resonant frequency of this material was not used, because the mechanical structure of the board was dampening this resonation extensive work is needed to optimise this movement board. A more thorough finite element Analysis using Comsoll or Ansys should be conducted using various parameters, materials and dimensions enabling us to gauge the best match of PZT more accurately to be used for different energy harvesting applications. An increase in the number and the size of the PZT plates is also needed to lower the resistance and higher the voltage which would mean higher amounts of power therefore allowing more scope to implement various loads.

\section{Acknowledgments}

The author would like to particularly thank Mr. Frédéric Pimparel of Morgan Technical Ceramics for the kind donation of PZT material.

\section{References}

[1] American Piezo LTD (2011). Piezo ELECTRIC CERAMICS: Principles and Applications. 2nd ed. Mackeyville, PA: APC Internationsl LTD. 1 - 114.

[2] David Darling, (2006). Hysteresis and Hysteresis Loops. Available: http://www.daviddarling.info/ encyclopedia/H/hysteresis_loop.html. [Accessed 04/02/2013]

[3] Department of Health. (2013). Be Food Smart' campaign 1 launches. Available: http://www.dh.gov.uk/health/2013/01/be-food-smart/. [Accessed 10/01/2013]

[4] Paul O'Shea, (8/08/20912) the electronic journal: time for change, available from: www.EEEtimes.com [Accessed September - October 2012]

[5] Vicor. (2013). Under voltage/Overvoltage Lockout. Available: http://cdn.vicorpower.com/documents/ application_notes/UV_OV_APPNote.pdf. [Accessed 12/03/2013]

[6] Stan Meyer. (2006. Hydro Cars. Available: http://tesla3.com/free_websites/ wf_meyer_hydrocars.html. [Accessed April 2013]

[7] A.B.Gill (1984). Power Plant Performance. $2^{\text {nd }}$ ed. London, UK: Butterworth and Co.

[8] Department of Health. (2013). Be Food Smart' campaign launches.Available: http://www.dh.gov.uk/health/2013/01/be-food-smart/. Last accessed 10/01/2013

[9] Paul O'Shea, (8/08/20912) the electronic journal: time for change, available from: www.EEEtimes.com [accessed September - October 2012]

[10] Morgan Electro Ceramics, 2007. Piezoelectric Ceaamics Catalogue. [Data Sheet] Ruabon, Wrexham MorganTechnical Ceramics. Available at: http:/www.morgantechnicalceramics.com/about-us/ [02/04/2013]

[11] Liner Technology, 2010. LTC3588. [Data Sheet] Milipitas,CA: Linear Technology. Available at: http://cds.linear.com/docs/en/datasheet/35881 fa.PDF [10/03/2013]

[12] Liner Technology, 2010. LTC3588. [Data Sheet] Milipitas,CA: Linear Technology. Available at: http://www.linear.cpm/product/LTC3588-1\#demoboards [10/03/2013]. 